\title{
Bilan scientifique du colloque « Transport Solide et Morphodynamique des Rivières » (TSMR2017) Villeurbanne, 8 et 9 novembre 2017
}

\author{
Benoît CAMENEN ${ }^{1}$
}

${ }^{I}$ Irstea, UR HHLY, centre de Lyon-Villeurbanne, 5 rue de la Doua, CS 20244, F-69625 Villeurbanne Cedex, France - e-mail: benoit.camenen@irstea.fr

\begin{abstract}
RÉSUMÉ. - Le colloque TSMR2017 a réuni plus de 100 participants principalement de France mais aussi de Suisse et de Belgique autour de 37 présentations orales dont deux conférences invitées et 25 posters. Cet article présente une synthèse des principaux éléments exposés lors de ce colloque autour des trois grands thèmes proposés : le transport solide en rivière, la morphodynamique des lits, et la gestion des sédiments et l'aménagement des cours d'eau.
\end{abstract}

Mots-clés : TSMR2017, transport solide, morphodynamique des rivières, gestion sédimentaire

\section{Scientific conclusion of the symposium on sediment transport and river morphodynamics (TSMR2017), Villeurbanne, 8-9th November, 2017}

\begin{abstract}
The TSMR2017symposium gathered more than 100 participants mainly from France but also from Switzerland and Belgium. 37 oral presentations including two keynote lectures were proposed together with 25 posters. A synthesis of the symposium is presented here including main issues from the three themes proposed: sediment transport, river bed morphodynamics, and sediment and river management.
\end{abstract}

Key-words: TSMR2017, sediment transport, river morphodynamics, sediment management

\section{INTRODUCTION}

Le transport solide et l'évolution morphologique des rivières constituent des thématiques centrales du fonctionnement des systèmes fluviatiles autant du point de vue fondamental qu'appliqué. La recherche fondamentale s'intéresse principalement aux processus hydro-sédimentaires, à leur conceptualisation, à la quantification des flux, à l'archivage des sédiments et à l'évolution morphologique des rivières. Le domaine appliqué montre parallèlement un besoin croissant de connaissances de l'impact des ouvrages sur les flux solides, de l'incision des lits, de la gestion des sédiments, etc. Ces questionnements ont amené des communautés variées à s'intéresser au fonctionnement physique des cours d'eau. Les méthodes employées sont très différentes et s'avèrent riches d'enseignements lorsqu'elles sont croisées :

- observations et mesures in situ ;

- recherche et modélisation en laboratoire ;

- modélisation numérique et analytique.

L'objectif du colloque TSMR 2017 a été, dans la continuité du premier colloque organisé en 2012 à l'université de Tours par Stéphane Rodrigues, de favoriser les échanges entre les différentes communautés francophones travaillant sur le transport solide, l'évolution morphologique et la dynamique physique des cours d'eau sur l'ensemble du continuum sédimentaire.

\section{COMITÉS}

Le comité local d'organisation de ce colloque s'est constitué sous la direction de Benoît Camenen avec l'aide précieuse des équipes «Hydraulique des rivières » (A. Paquier, C. Berni, M. Chatelain, T. Chibane, J.B. Faure, J. Le Coz, M. Launay, L. Pénard, E. Perret, S. Proust), et " Métrologie » (A. Buffet, G. Dramais, F. Thollet) et de l'appui à la recherche du centre d'Irstea Lyon (H. Faurant, V. Aburegaiba).

Le comité scientifique a aussi fortement contribué au succès du colloque avec la lecture des résumés: P. Belleudy (Université Grenoble Alpes), C. Berni (Irstea Lyon), B. Camenen (Irstea Lyon), A. Crave (Université Rennes), G. Dramais (Irstea Lyon), F. Gob (Université Paris 1), E. Lajeunesse (IPGP), J. Le Coz (Irstea Lyon), F. Liebault (Irstea Grenoble), J.-R. Malavoi (EDF), V. Mano (Artelia-Environnement), G. Nord (Université Grenoble Alpes), C. Peteuil (CNR), H. Piégay (CNRS - ENS Lyon), O. Radakovitch (CEREGE), A. Recking (IRSTEA Grenoble), S. Rodrigues (Université Tours), et M. Tal (CEREGE).

\section{SESSIONS}

Le programme du colloque s'est déroulé autour de deux séminaires invités et les trois thèmes proposés pour ce colloque :

- Transport solide en rivière (27 communications);

- Morphodynamique des lits (18 communications);

- Gestion des sédiments et aménagement des cours d'eau (15 communications).

Le livre des résumés, le programme détaillé, ainsi que l'ensemble des présentations (orales et poster, sous réserve d'autorisation ou mise à disposition par les auteurs) sont disponibles sur le site du colloque : https://tsmr2017.sciencesconf.org. 


\section{III.1. Séminaires invités}

Deux séminaires invités ont été proposés lors de ce colloque. Le premier séminaire par Alessandra Crosato (IHE Delft, Pays-Bas) portait sur les bancs en rivière alluviale. Une présentation didactique des différents types de bancs, de leurs processus de formation (forçage externe, instabilité), de leurs caractéristiques (système ponctuel, périodicité, mobilité, etc.), des approches par instabilité linéaire pour les modéliser (bancs libres ou forcés) a été proposée ainsi que des retours d'expérience combinant modélisation et expérience de laboratoire et terrain. Enfin, des réflexions sur la possible gestion de ces corps sédimentaires pour la navigation, la prévention du risque d'érosion des berges, et la diversité des habitats ont été discutées. Le second séminaire par Pierre Francus (INRS, Québec, Canada) a permis de faire le lien entre la dynamique des Matières en Suspension (MES), largement discutée au cours de ce colloque, et la sédimentologie. Plus particulièrement, une discussion a été présentée sur les enregistrements des flux sédimentaires et les possibles reconstitutions des débits et crues extrêmes sur le long terme (plusieurs dizaines voire centaines d'années) à partir d'analyses de sédiments lacustres annuellement laminés (appelés aussi varves). Il existe un nombre limité de sites où des varves sont présentes mais celles-ci par leur lamination caractéristique avec une différenciation nette des couches hivernales et estivales, permettent avec l'aide d'une instrumentation de pointe (microscope électronique à balayage, Itrax core scanner, CT-scanner) de reconstruire une paléohydrologie et quantifier les impacts du changement global.

\section{III.2. Transport solide en rivière}

Le thème sur le transport solide en rivière était le plus représenté sur ce colloque avec environ $50 \%$ des présentations et trois sous-thèmes : (i) les processus et modélisation (ii) les mesures in situ de la charge grossière (charriage et suspension), et (ii) les mesures des flux en MES et polluants associés. Plusieurs présentations ont porté sur des développements instrumentaux (acoustique passive pour le charriage : Zanker et al, Gassier et al. ; traceur actifs : Cassel et al. ; acoustique active pour la suspension : Vergne et al.). Plusieurs études de processus en laboratoire ont été présentées, principalement sur les effets des mélanges ou des formes de fond (Camenen et al., Dhont \& Ancey, Perret et al.). On peut aussi noter que la modélisation numérique est aujourd'hui un allié incontournable des études du transport solide (Alliau et al., Alliès \& Peret, de Linares et al., Piasny et al., Mano et al.) mais que les modèles physiques restent toujours d'actualité (Alliau et al., El Kadi Abderrezzak). Quelques cas d'étude ont été discutés allant des petites rivières de plaine (Gilet et al., Houbrecht et al., ) aux grands fleuves comme le Rhône (Vasquez-Tarrio et al.) ou la Loire (Le Guern et al.), en passant par les torrents de montagne (Blanpied et al., Vasquez-Tarrio et al.). Enfin, la dynamique des Matériaux en Suspension a été abordée principalement via les réseaux de mesure de turbidité (Camenen et al, Debret et al., Deloffre et al., Misset et al., Mengin et al.) mais aussi via l'échantillonnage des matériaux en suspension et des contaminants associés (Berni et al., Ferracci et al., Losson et al.), voire l'étude de la dynamique des dépôts (Paquier et al., Pénard et al.).

\section{III.3. Morphodynamique des lits}

Le thème de la morphodynamique des lits de rivière incluait deux principales échelles d'étude : (i) une échelle plus locale sur l'événementiel avec la dynamique des formes de fond (dunes, bancs, etc.) et (ii) une échelle plus globale sur le long terme avec les évolutions récentes et passées des lits fluviaux et la continuité sédimentaire. Concernant le second point, des exemples divers et couvrant le territoire français ont été montrés, allant de la Réunion (rivière des Galets : Peteuil \& Nassor), la Nouvelle Calédonie (Bertrand \& Liébault) au Sud-Ouest (Garonne : Jantzi et al. ; Dordogne : Bulteau et al.), au Centre et à la Normandie (Carbon et al., Lacoste et al.) mais aussi à l'étranger avec les exemples de l'Oubangui au Congo (Nzango et al.), de l'Ehen en Angleterre (Marteau et al.) ou de rivières néo-zélandaises (Le Coz et al.). D'un point de vue technique, ce colloque a aussi montré la montée en puissance de la télédétection à partir d'imagerie aérienne (Bertrand \& Liébault, Coutaz et al.), satellite (Belletti et al.) et LiDAR (Chapuis \& Ait Elabas). On pourra d'ailleurs noter que le prix du meilleur poster a été attribué à Jonathan Coutaz et collaborateurs pour leur étude intitulée "Méthodologie d'obtention de MNS Topo-bathymétrique à partir de photogrammétrie ». La dynamique des barres sédimentaires a été abordée principalement via l'impact de la végétation (Jourdain et al., Wintenberger et al.) mais aussi via leur impact sur le transport solide (Dhont et Ancey).

\section{III.4. Gestion des sédiments et aménagement des cours d'eau}

La gestion des sédiments et l'aménagement des cours d'eau est une thématique pour laquelle les contributeurs venaient principalement des grands gestionnaires de rivières (EDF, CNR) et des bureaux d'étude. Trois sujets principaux ont été évoqués : (i) l'altération de la continuité sédimentaire, (ii) les dépôts sédimentaires potentiellement contaminés, et (ii) la restauration physique des rivières. Plusieurs présentations avaient ainsi pour sujet le suivi des impacts de seuils ou barrages sur les flux sédimentaires et leur gestion, en particulier sur le Rhône avec la gestion des chasse du Haut-Rhône (Pollin et al.) mais aussi des suivis de sites particuliers (Frétaud et al., Reynaud \& Taisant). Plusieurs analyses ont aussi été présentées sur de petits cours d'eau (Landon, Reulier et al., Thomas \& Tiriau). En terme de restauration, des cas de redynamisation du lit par lâcher d'eau claire ont été introduits (Landon et al., Loire et al.). De nombreux exemples de restauration hydromorphologique de tronçon de rivière ont de plus permis de voir la variété de solutions mais aussi les nombreuses difficultés de mise en place de ces restaurations et surtout de leur suivi sur le long terme (Chardon et al., Handfus et al., Laval et al., Rodrigues et al., Yassine et al.).

\section{CONCLUSIONS}

Cette seconde édition du colloque a permis de confirmer la richesse des études mais aussi des techniques utilisées pour la meilleure compréhension des flux solides et des évolutions morphologiques dans un contexte de rivières fortement anthropisées. On peut observer une dynamique très forte de notre communauté francophone, que ce soit pour le développement méthodologique mais aussi la mise en place de restauration et gestion de rivières complexes. Les échanges ont ainsi été très fructueux entre différentes communautés qui n'échangent pas nécessairement assez : académiques et ingénieurs, géographes et hydrauliciens... Ceci montre bien l'intérêt d'échanger et partager nos savoirs scientifiques et techniques. Ces discussions peuvent être prolongées grâce à 
la liste de diffusion morphosed (morphosed@lists.irstea.fr ; contacter M. Jodeau, J. Le Coz ou B. Camenen pour l'adhésion) qui permet d'échanger sur la thématique (questions scientifiques, proposition de stage ou thèse, etc.) et inclue aujourd'hui 165 adhérents. En lien étroit avec cet objectif de rapprocher les différentes communautés sur cette thématique, il nous a semblé important de rendre ce colloque accessible en limitant au maximum les frais d'inscription. Ceci a pu être possible grâce à nos sponsors que nous tenons à remercier. Le succès d'un tel colloque montre clairement l'intérêt grandissant de cette thématique en France et ailleurs, et donc l'intérêt de maintenir ce type d'événement de façon régulière. Rendez-vous donc en 2020 pour la troisième édition !

\section{REMERCIEMENTS}

L'ensemble de nos sponsors et partenaires qui ont permis l'organisation optimale de ce colloque sont remerciés : EDF, CNR, Eau du Grand Lyon, la région Auvergne-Rhône-Alpes (dont l'ARC Environnement), Irstea, AFB, et SHF.

\section{SHE}

Manifestations 2018

- 2018, mars, Grenoble Glaciologienivologie- hydrologie de montagne

- 2018, 28 février-2 mars, Strasbourg: $\mu$ Flu'18 -NEGS'18

- 2018, 7 juin, Marseille : dessalement et adaptation aux changements climatiques?

- 2018, septembre, Toulouse : séminaire Ecoulements diphasiques

- 2018, 14-16/11 : De la prévision

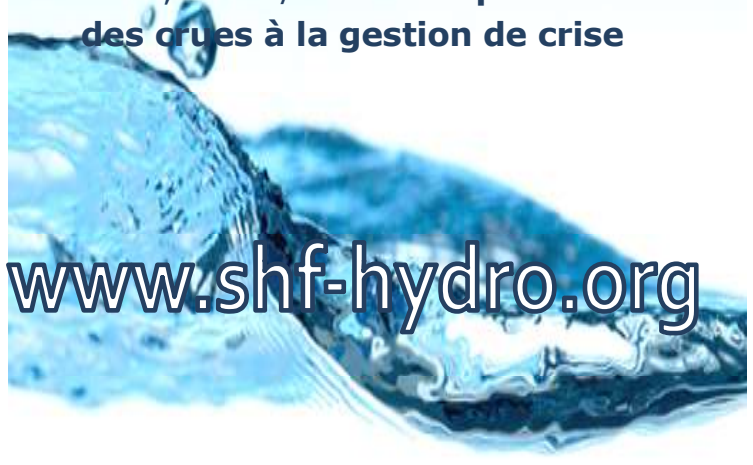

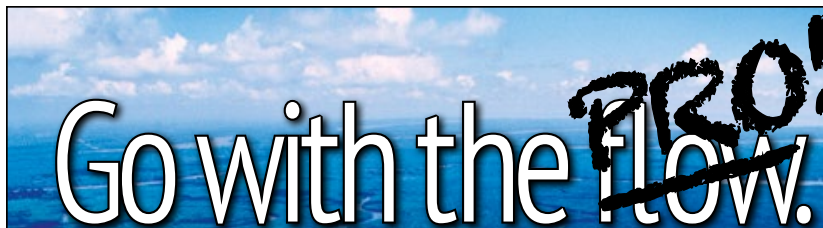

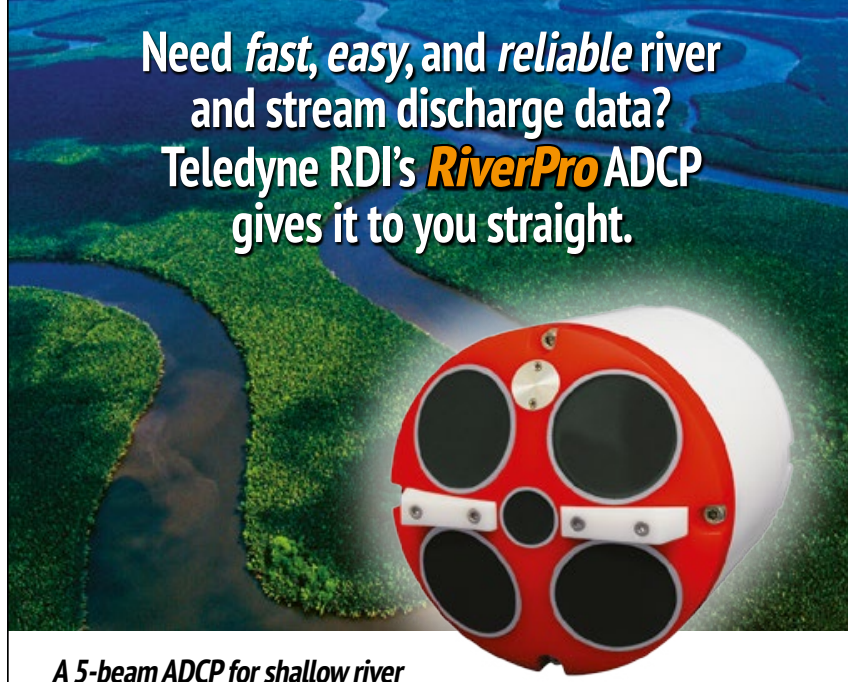

A 5-beam ADCP for shallow river

environments, RiverPro delivers the simplicity and reliability your operations require, at a price that meets your budget. Learn more at: http://rdinstruments.com/product/adcp/riverpro-adcp

RiverPro... right on $\underline{Q}$
TELEDYNE

RD INSTRUMENTS Everywhereyoulook" www.rdinstruments.com 